\section{Nature's magic bullets}

\author{
Antibiotics: Actions, Origins, \\ Resistance \\ by Christopher Walsh \\ ASM Press: 2003.335 pp. \$99.95; \$89.95 \\ to members of the American Society for \\ Microbiology

\section{Stewart T. Cole}

Starter units, expansion, modification, mergers, restructuring, down-sizing and cleavage. There's nothing new there today's pharmaceutical industry is merely following the business plan laid down by nature for the production of antibiotics by microbes. In the face of market forces, the creation of giant pharmaceutical companies that concentrate extensive resources on lifestyle diseases, at the expense of the research and development (R\&D) of new anti-infective agents, is giving justifiable grounds for concern. In this book, devoted exclusively to antibacterial agents, Chris Walsh provides an authoritative account of their (bio)synthesis, mode of action and resistance mechanisms, and highlights new strategies to find and improve antibiotics. This latter topic should prove to be a source of inspiration for the R\&D directors of big pharmaceutical companies.

The first of the book's five sections outlines the concepts to be developed later. It highlights the slow pace of development of new agents in recent decades. The second section introduces the main classes of antibiotic that target cell-wall biosynthesis, the metabolism of nucleic acids, the proteinproducing machinery and the folic acid pathway. Like the rest of the book, it is superbly illustrated: several colour plates take full advantage of the remarkable progress in structural biology to show, for instance, how macrolide antibiotics bind to the polypeptide exit tunnel of the ribosome and prevent proteins from being made.

The third section concentrates on the principal resistance mechanisms that block the action of antibiotics on pathogens and relates how these are commonly used by the antibiotic-producing organisms to protect themselves from their own products. These mechanisms include modifying the target, destroying the antibiotic, and lowering its concentration to ineffective levels. The importance of active efflux to antibiotic resistance is also given due attention, although the trick of altering cell permeability to reduce the entry of antibiotics is only addressed in a cursory manner. Parts of the book would have benefited from critical appraisal by a microbiologist, but this is a minor quibble.

In a fourth section that is somewhat

\section{Online database}

\section{Island investigations}

More than a hundred European scientific expeditions went to the Canary Islands during the eighteenth and nineteenth centuries. Many were on their way to more distant destinations - but others were there to map and study the natural history of the islands themselves, and led to many scientific discoveries of general importance.

The original reports of these expeditions, physically scattered around the world, are now being brought together courtesy of a major European Commission-funded digital project known as ECHO (European Cultural Heritage Online).

Some of the works have already been widely seen. The 1856 expedition of Charles Piazzi-

Smith, for example, was recorded in his popular work An Astronomer's Experiment (1858), held in several libraries in Spain and Britain. PiazziSmith made astronomical observations at different altitudes on El Teide, Tenerife's highest peak, showing for the first time that more stars can be seen at the top of a mountain.

By contrast, the work of the Norwegian

anthropocentric in its reasoning, we are introduced to the molecular rationale that governs the assembly-line production by actinomycetes and fungi of antibiotics belonging to the polyketide and nonribosomal peptide classes. We encounter cascades of enzymes and massive protein factories that, following the active growth phase, manufacture secondary metabolites, many of which act as antimicrobial agents or immunosuppressors. The environmental cues and regulatory effects of the signaltransduction networks that govern the expression of the corresponding genes are also explained, but insufficient attention is paid to the forces of natural selection. However, Walsh lucidly expounds how genomics, biochemistry and structure determination allow one to predict the composition of a polyketide if you know the arrangement of the catalytic domains of the enzyme responsible for its synthesis; he implies that the reverse prediction is also feasible.

This sets the tone for the fifth and final section, which elegantly relates how new antibiotics can be produced from existing compounds or biosynthetic pathways. It also explains why engineering polyketide synthases, and other enzymes that produce or modify antibiotics, will enable us to generate in a rational manner new agents that could have therapeutic value.

The strength of the book lies in Walsh's mastery of chemistry and his ability to explain complex (bio)chemical reactions in a simple yet vivid fashion. The reader will botanist Christen Smith, who accompanied German volcanologist Leopold von Buch in his 1814 expedition, was thought to be lost, as Smith continued on to the Congo where he died. But his manuscripts and diaries, describing some 600 different plants, about 50 of which were new to science at the time, were recently found in a library in Oslo.

The picture shows illustrations of volcanoes taken from Atlas des Isles Canaries (1836) by von Buch, who developed his theory of volcanoes in the Canaries.

http://humboldt.mpiwg-berlin.mpg.de

conclude, correctly, that most of our clinically useful antibiotics stem from research on microbes, and that, with just a few exceptions, synthetic and combinatorial medicinal chemistry have yet to achieve their full potential. Nonetheless, Paul Ehrlich, Alexander Fleming and Selman Waksman would all have found something to enjoy in Antibiotics.

Stewart Cole is in the Unité de Génétique Moléculaire Bactérienne, Institut Pasteur, 28 rue du Docteur Roux, 75724 Paris, France.

\section{Light summer reading}

Isaac Newton

by James Gleick

Pantheon, £22 (also Fourth Estate, £15,

available in September)

An acclaimed new biography of one of the fathers of physics.

\section{Einstein}

by P. D. Smith

Haus, £12.99 (hbk), £8.99 (pbk)

A new concise biography of the famous physicist.

\section{Lovelock and Gaia}

by Jon Turney

Icon, £9.99

A brief account of James Lovelock's vision of a self-regulating planet and his influence on Earth system science. 